\title{
FLOW DYNAMICS OF WAVES PROPAGATING OVER DIFFERENT PERMEABLE BEDS
}

\author{
Corvaro Sara ${ }^{1}$, Mancinelli Alessandro ${ }^{2}$ and Brocchini Maurizio ${ }^{3}$
}

\begin{abstract}
The analysis of the hydrodynamics over porous media is of interest for many coastal engineering applications as the wave propagation over permeable structures or gravel beaches. The study of a boundary layer evolving over permeable beds is important to a better understanding of the interactions between the flow over and inside the porous medium. An experimental study has been performed to analyze the dynamics produced when waves propagate over two kinds of permeable beds: spheres (regular permeability) and natural stones. For comparative purposes the same analysis has been extended to two rough beds made, respectively, by a single layer of spheres and natural stones. We here focus on the correlation between the wave energy reduction induced by a porous bed and the flow resistance. An experimental law for the prediction of the friction factor is found by using the log-fit method in analogy to that reported in Dixen et al. (2008) for rough beds. Moreover, inspection of the turbulent velocity components allows one to evaluate the bottom shear stress. The latter analysis has been performed for different permeable beds (regular and irregular beds). A good agreement between the bottom shear stress behavior and the wave height attenuation over rough and permeable beds (Corvaro et al. 2010 and Corvaro et al. 2014a, 2014b) has been observed.
\end{abstract}

Keywords: permeable bed, porous medium, wave damping, friction factor

\section{INTRODUCTION}

The interactions between the flows propagating in a free-fluid region and those restrained by the resistance of a porous medium are of interest for many coastal engineering applications. The interaction of sea waves with either permeable structures (e.g. Losada 1996) or a porous seabed (e.g. Hsiao et al. 2002) is a classic problem of coastal engineering: the elevation of the water table and its influence on groundwater infiltration/exfiltration processes within the swash zone may contribute to accretionary or erosional trends at the shoreline. Some artificial systems use such beach capability by means of a drainage in order to increase the permeability of sand favoring the settlement of the suspended sediment load on the beachface during the uprush phase and contrasting its offshore movement during the backwash phase (Damiani et al 2010, 2011).

Over the years numerous experimental, theoretical and numerical studies have been performed to characterize the damping of water waves over a porous medium. Fundamental for the understanding of the nearbed wave flows are the experimental studies on oscillatory boundary layers occurring over both smooth (e.g. Nielsen 1992) and rough (e.g. Sleath 1987) beds. During recent years the study of turbulent boundary layers on a porous bed has received much attention and provided much information about the hydrodynamics over and inside the porous medium. Numerical models are most often based either on the Nonlinear Shallow Water Equation (e.g. Wurjanto and Kobayashi 1993; Van Gent 1995) or on Boussinesq-type equation (e.g. Cruz et al. 1997) or on the Reynolds Averaged Navier Stokes equations (e.g. Karunarathna and Lin 2006).

The wave energy dissipation rate $\varepsilon_{p}$ due to a permeable bed has been evaluated by analyzing the kinematics on the bottom boundary layer. In particular the paper focus on the experimental evaluation of the mean and turbulence properties of the bottom boundary layer, on the evaluation of the friction factor and, hence, on the shear stress produced over different types of bed with large roughness. A study similar to the present analysis is the experimental investigation of Lara et al. (2002), which analyzed wave flows on a porous bed by means of a PIV technique. In the same fashion, we have here used a different non-intrusive technique (i.e. the Particle Tracking Velocimetry). The mean velocity profiles, measured at different phase values, were displayed in semi-log plots in order to obtain both the bottom friction velocity $u^{*}$ and the equivalent sand roughness $\mathrm{k}_{\mathrm{s}}$ from which the wave dissipation due to porous bed can be evaluated. As the flow propagates through the porous medium, resistances act on the fluid due to both fluid viscosity and fluid-particle interactions. The wave height attenuation (Corvaro et al. 2010) and the wave velocity reduction (Corvaro et al. 2014a) were analyzed in previous

\footnotetext{
${ }^{1}$ DICEA, Università Politecnica delle Marche, Via Brecce Bianche, 1, 60131, Ancona, Italy, s.corvaro@univpm.it

${ }^{2}$ DICEA, Università Politecnica delle Marche, Via Brecce Bianche, 1, 60131, Ancona, Italy, a.mancinelli@univpm.it

${ }^{3}$ DICEA, Università Politecnica delle Marche, Via Brecce Bianche, 1, 60131, Ancona, Italy, m.brocchini@univpm.it
} 
works. Burcharth and Andersen (1995) and Van Gent (1995) discussed in detail the role of the different friction parameters which influence the flow through a porous medium. Our experiment adds information to that already available because of the broad analysis of the wave dissipation phenomenon influenced by a wider range of wave characteristics.

\section{EXPERIMENTAL SETUP}

The experimental tests were performed at Hydraulics Laboratory of the Università Politecnica delle Marche (Ancona, Italy). The channel is $50 \mathrm{~m}$ long, $1 \mathrm{~m}$ wide and $1.3 \mathrm{~m}$ high. The wave motion is forced by a piston-type wavemaker. The wave motion is forced by a piston-type wavemaker. An absorbing mildly sloping beach (slope 1:50) made of gravel, was used to reduce the wave reflection and to guarantee an undisturbed flow in the measuring area. The sidewalls of the flume are glassed for the central $36 \mathrm{~m}$ and enable one to film and carry out optical measurements.

A reduced geometrical scale of 1:10 has been used in the physical model Moreover, the wave characteristics were reproduced by using Froude similarity. In Figure 1 is shown the wave channel with the physical model location. The reference system is reported in Figure 2. Regular monochromatic waves with different wave heights $H_{i}(5 \mathrm{~cm}-10 \mathrm{~cm})$ and wave periods $T(1.5-2.5 \mathrm{~s})$ were run and forced to propagate over the physical model. In Table 1 the wave characteristics over the physical model were summarized, where $H_{i}$ is the wave height at the upstream section of the physical model, $h$ and $L$ are, respectively, the water depth and the wave length over the physical model and $U r$ is the Ursell parameter expressed as $U r=H_{i} L_{2} / h^{3}$.

The physical model is $1.5 \mathrm{~m}$ long, although more significant damping would be achieved by using a longer porous bed (Corvaro et al. 2010), the purpose of the present analysis is that of characterizing typical dynamics of nearshore waves evolving over dissipative seabeds. Breaking waves were not considered because the surface-generated turbulence could obscure too much the whole dynamics. Waves were generated on a depth of $0.47 \mathrm{~m}$ (depth at the wave paddle), then propagated on a ramp with slope $1: 15$, up to the water depth $h$ of $0.30 \mathrm{~m}$. Once arrived on the horizontal bottom, waves run on an impermeable bed (made of steel plates above wood platforms), then on the physical model.

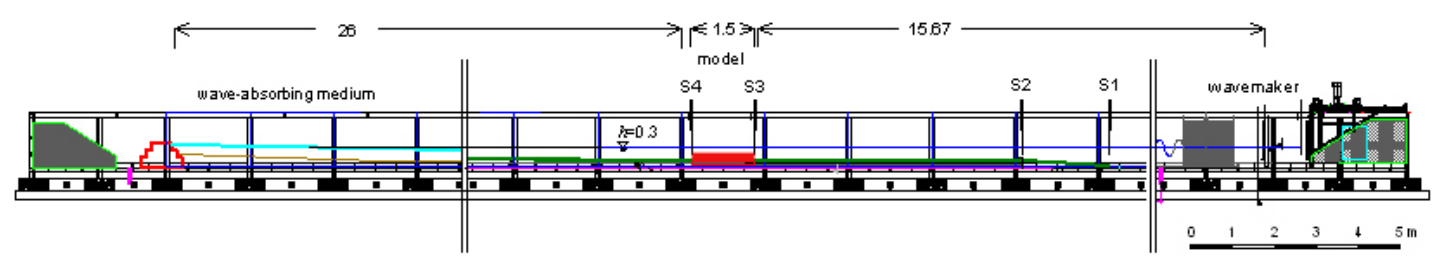

Figure 1 - Wave channel with the physical model location.

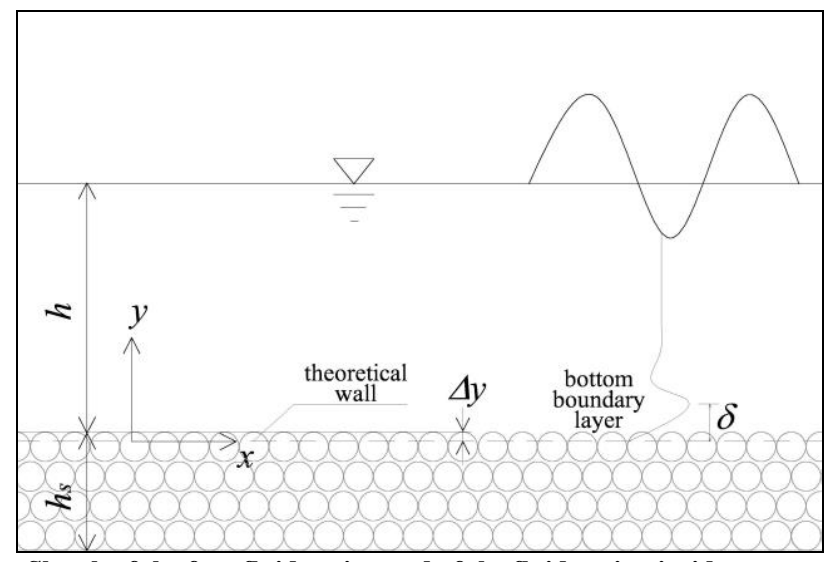

Figure 2 - Sketch of the free-fluid region and of the fluid region inside a porous medium 


\begin{tabular}{|lcccr|}
\hline \multicolumn{5}{|c|}{ Table 1 - Wave characteristics over the physical model } \\
\hline Wave & $H i(\mathrm{~cm})$ & $T(\mathrm{~s})$ & $h(\mathrm{~m})$ & $\mathrm{Ur}(-)$ \\
\hline OR2 & 4.4 & 1.5 & 0.30 & 9 \\
OR3 & 4.5 & 2.0 & 0.30 & 18 \\
OR4 & 8.5 & 2.5 & 0.30 & 54 \\
OR5 & 9.3 & 1.5 & 0.30 & 19 \\
OR6 & 9.6 & 2.0 & 0.30 & 38 \\
OR10 & 11.6 & 2.5 & 0.30 & 74 \\
\hline
\end{tabular}

Different bed configurations (see Figure 3) were tested: rough impermeable beds and permeable beds. Both the rough impermeable and the permeable beds were made of either plastic spheres (diameter $D_{50}=3.6 \mathrm{~cm}$ ) or natural stones (diameter $D_{50}=2.85 \mathrm{~cm}$ ). In particular, the rough beds were made of a single layer, while the porous medium built with more layers of spheres or natural stones for a total thickness of $12.1 \mathrm{~cm}$. The regular permeable bed is made by four layers of plastic spheres arranged in a rhombohedral shape, this providing a porosity $n=0.29$. The stones were arranged in a random orientation with a dense packing with a porosity $n=0.30-0.35$. In Figure 3 are summarized the two types of permeable beds tested in such an experimental campaign.

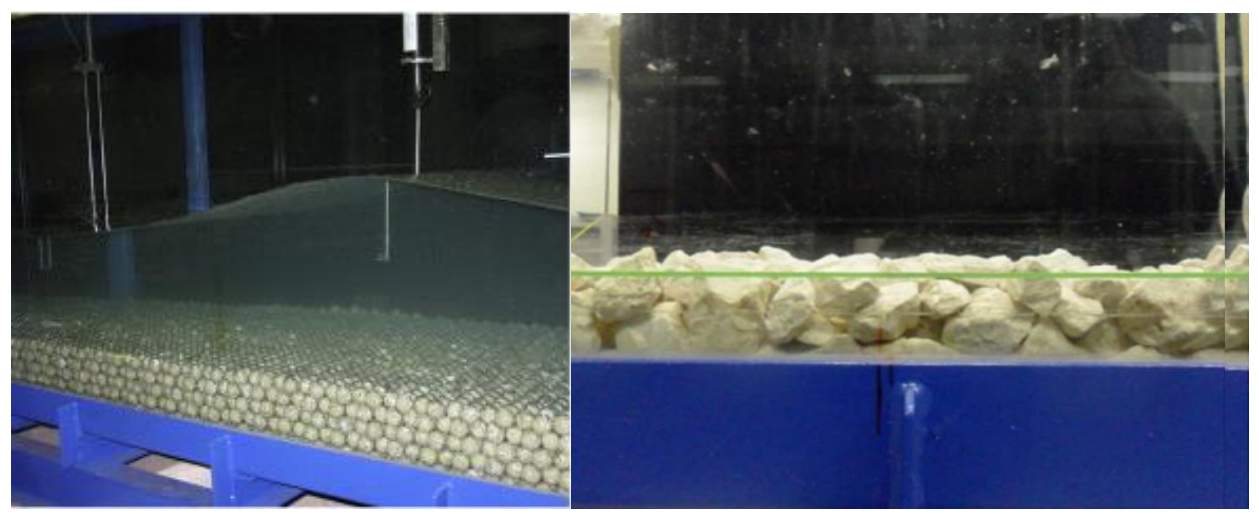

Figure 3 - Different permeable beds: spheres (left) and natural stones (right).

Six electro-resistive wave gauges were used along the flume to measure the instantaneous flow level. The elevation gauges, placed over the physical model, were called "S3 and "S4" placed, respectively, $0.15 \mathrm{~m}$ and $1.35 \mathrm{~m}$ downstream of the upstream section of the physical model (see Figure $4)$.

The region over the physical model (FOV) was illuminated and video-recorded, with the purpose to reconstruct the velocity field at a later stage (see Figure 4). Three halogen lamps generated a light sheet with thickness of $1 \mathrm{~cm}$ along the central streamwise section of the flume. A digital CCD camera (Dalsa Falcon 4M60), with resolution of 2352x1728 pixels and resolution depth of 8bit, ensured the recording of a $30 \times 22 \mathrm{~cm}^{2}$ vertical window over the porous bed, with an image pixel size of about 13 $\mu \mathrm{m}$. In particular, a frame rate of 50fps was used for the present experiments. Particles of ground chili pepper, with median diameter of $0.3 \mathrm{~mm}$ and density $\rho=1000 \mathrm{~kg} / \mathrm{m} 3$ were used as neutral seeding.
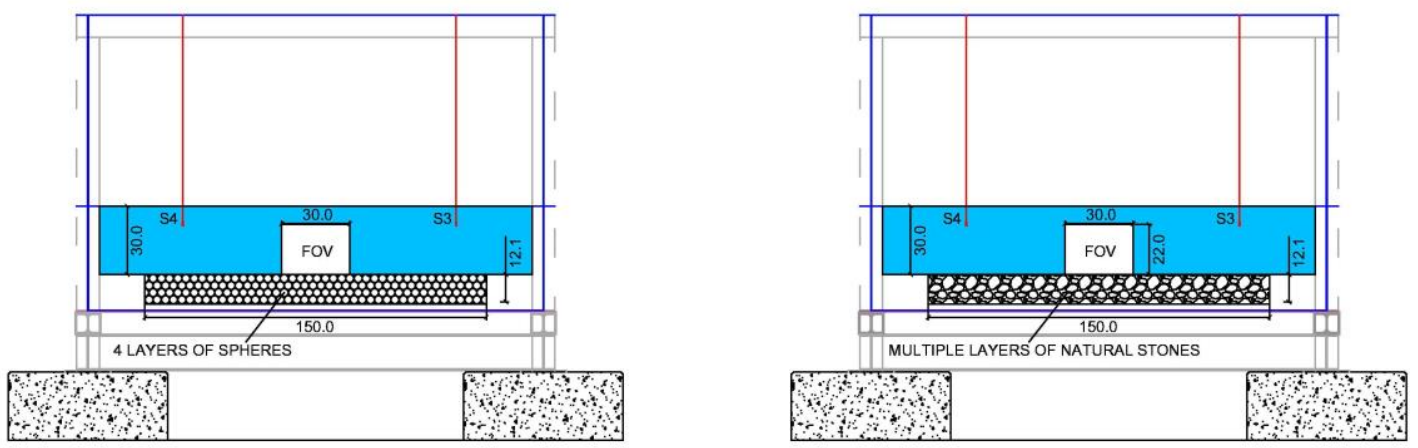

Figure 4 - Location of the region over the physical model for the different permeable beds: spheres (left) and natural stoned (right). 


\section{VELOCITY DATA ANALYSIS}

To study the mean flow and the turbulence produced by non-breaking waves over, the time series of horizontal $(u)$ and vertical $(v)$ velocity components were elaborated to extract the mean and fluctuating terms from the instantaneous data. For sake of brevity we report the analysis only for the horizontal velocity component. To have a good representation of the turbulence we analyze more than 80 waves ( $N$ is the number of the waves of each realization). For a stationary phenomenon the average over the time span $\tilde{T}$ is defined:

$$
\bar{u}=\frac{1}{\tilde{T}} \int_{t}^{t+\tilde{T}} u(t) d t
$$

and the fluctuations were calculated as the difference between the instantaneous and mean data indicated by the overbar:

$$
u^{\prime}=u-\bar{u}
$$

For a periodic signal, due to the deviations of the wave signals from a perfectly periodic, symmetric and repeatable form, the fundamental wave phases (crest, trough, nodes) did not occur exactly at the same instant in the wave cycles. To obtain values that express correctly the statistics at such phases, we used also the so-called phase average $\langle>$ that consists in fixing the phase $(\omega t=\hat{\varphi}$, where $\omega$ is the angular frequency of the wave) and averaging arithmetically all the "waves" of each realization:

$$
<u(\hat{\varphi})>=\frac{1}{N} \sum_{n=1}^{N} u_{n}(\hat{\varphi})
$$

where the subscript $n$ indicates the $n$th wave of each realization.

The phase-averaged values of the turbulent variables are defined as follows:

$$
\left\langle u^{\prime}(\hat{\varphi})\right\rangle=\sqrt{\left\langle u^{\prime}(\hat{\varphi})^{2}\right\rangle}=\sqrt{\frac{1}{N} \sum_{n=1}^{N}\left(u_{n}(\hat{\varphi})-<u(\hat{\varphi})>\right)^{2}}
$$

\section{RESULTS AND DISCUSSION}

The wave energy flux $E C_{g}$, the product of the total mean wave energy per unit area $E$ and wave group celerity $C_{g}$, is dissipated by a number of mechanisms, as described by:

$$
\frac{\partial E C_{g}}{\partial x}=-\varepsilon_{b}-\varepsilon_{f}-\varepsilon_{p}
$$

where the dissipative contributions due to wave breaking, bottom friction, and bed permeability are, respectively, $\varepsilon_{b}, \varepsilon_{f}$, and $\varepsilon_{p}$. In Postacchini et al. (2011) is reported a comparative analysis on the wave energy dissipation efficiency on various coastal protection devices.

The determination of the dissipation rate due to a permeable bed has been investigated by means of the analysis of the boundary layer dynamics. The flow resistance in the internal flow is characterized by the wave friction factor $f_{w}$. The evaluation of $f_{w}$ is important to correlate the behavior of the bottom boundary layer and the wave damping that occurs when flow propagates over porous beds.

The shear stress has been evaluated both through the log-fit method and by means of the turbulent components $\left\langle-u\right.$ ' $\left.v^{\prime}\right\rangle$. From the knowledge of the friction factor $f_{w}$, the maximum bottom shear stress can be calculated as $\tau_{\max }=0.5 f_{w} \rho U_{\text {OMax }}^{2}$, where $U_{\text {OMax }}$ is the undisturbed flow velocity 


\section{Log fit-method}

The log-fit method has been used in the literature for the evaluation of the shear stress induced by rough beds. Authors applied such a method with different procedures. Dixen et al. (2008) proposed one to determine from semi-logarithmic plots of the velocity distributions, the bed friction velocity $u_{*}$ and the equivalent bed roughness $k_{s}$, when the boundary layer is thick enough to accommodate the logarithmic layer in it. In the literature such a procedure has been applied only for rough bed. The position of the "true seabed" $\Delta y$ is usually at about $0.2 D_{50}$, being $\Delta y$ the distance between the top of the spheres (or stones) and the theoretical bed (see Figure 2). For example Dixen et al. (2008) suggested that the "true seabed" be placed at $0.23 D_{50}$ below the top of the ping-pong ball that they used, that was with the same diameter of ours spheres.

This procedure consists, essentially, in:

i)plotting, at different phases, the mean horizontal velocity vertical profiles in a semi-logarithmic graph;

ii) setting an arbitrary position of the "true seabed" (the vertical $y$-axis points from the "true seabed" upwards, as shown in Figure 2);

iii) identifying the interval $0.1 D_{50}-0.2 D_{50}<y<0.3 \delta$ for which the straight-line portion exists (where $\delta$ is the boundary layer thickness defined as the limit where the upper overshooting occurs, as shown in Fig. 3, for the analyzed phase). The upper limit ensures that the $y$ level is in the constant stress layer, while the lower limit ensures that the velocity variation is not influenced by the bottom roughness (Grass 1971),

iv)determining the friction velocity $\left(u_{*}\right)$ from the slope of the straight line and the equivalent obstacle roughness $\left(k_{s}\right)$ from its intercept with the $y$-axis (through the logarithmic law $\langle u\rangle=(u * /) \log \left(30 y / k_{s}\right)$, where $\kappa$ is the von Karman constant $)$.

Here, our purpose is to apply the same procedure to a permeable bed for the determination of the equivalent bottom roughness $k_{s}$ and the bottom friction velocity $u *$ from which it can be obtained the wave friction factor $f_{w}$ as:

$$
f_{w}=2\left(\frac{u_{*}}{U_{0 \max }}\right)^{2}
$$

where $U_{0 \max }$ is the undisturbed velocity far from the bottom boundary layer.

The behavior of wave boundary layer is modified when waves propagates over a permeable bed: at the interface between the external and internal flows the vertical velocity (discharged velocity) is not equal to 0 , because a flux of the flow between both layers is produced. However, by analyzing the vertical profiles of the horizontal velocity over a permeable bed, the typical behavior of wave-forced bottom boundary layers with velocity overshootings at the interface with the free-stream region and maximum value at the wave crest phase has been observed. Therefore, it is observed the validity of a logarithmic law within the bottom boundary layer for phases with non-vanishing horizontal velocity.

The procedure is delicate as already revealed in the literature (e.g. Justesen 1988) and a uniform application for a very large different wave characteristics (in our experiments $U r$ changes from about 10 to 80 ), for a small values of the roughness parameter $A / k_{s}$ (the roughness is large with respect to the the oscillation amplitude $A$ ) and for different bed configurations has been not simple to find. Therefore a preliminary comparison of the velocity profile for three different bed configurations has been made. In Figure 5 and Figure 6 are reported the non-dimensional horizontal velocity profile $\langle U\rangle / H_{i} \omega$ at the crest wave phase versus the non-dimensional depth $y_{0} / h$ (where $y_{0}$ is the vertical upward distance from the top of the sphere: $y_{0}=y-\Delta y$ ) for, respectively, waves of period $T=1.5 \mathrm{~s}$ (OR2 and OR5) and $T=2.0 \mathrm{~s}$ (OR3 and OR6).

It can be noticed that the velocity profiles change with the bed configurations, even if a logarithmic layer has been always observed also over a permeable bed. In particular it is found that within the bottom boundary layer the horizontal velocity over the permeable bed becomes larger than those over a rough bed and, generally, displays a larger peak, demonstrating that the velocity goes to zero for lower value of $y$. Therefore, for a permeable bed the position of the theoretical bed can be placed at a deeper location with respect to a rough bed. 

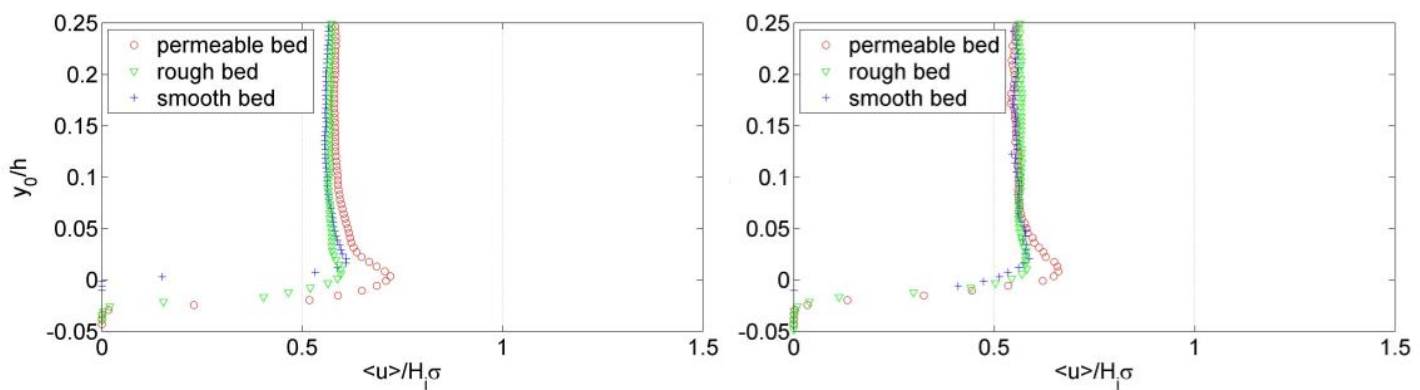

Figure 5 - Horizontal velocity profile for different bed configurations: smooth bed (blue); rough bed (green); permeable bed (red). OR2 (left) and OR5 (right)
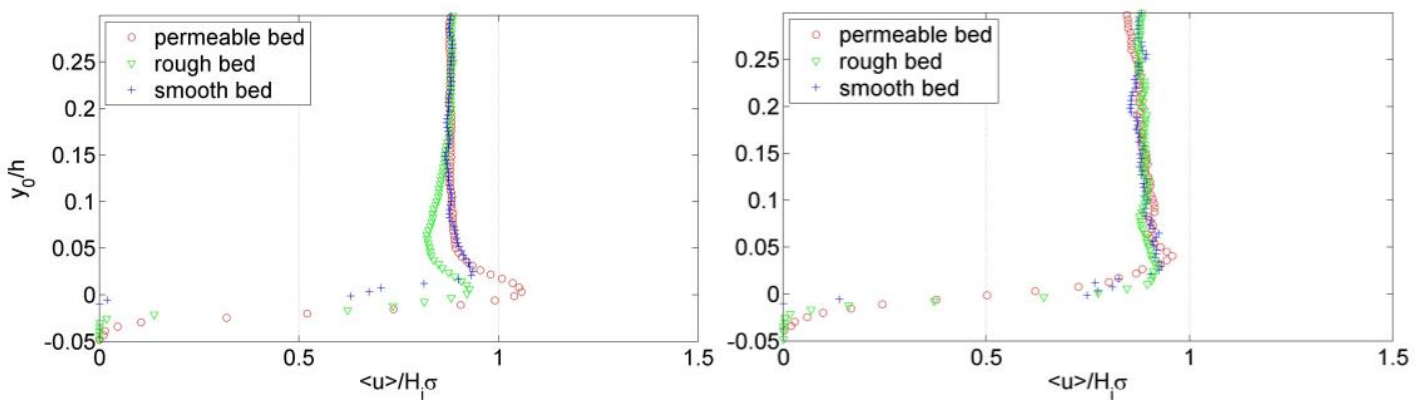

Figure 6 - Horizontal velocity profile for different bed configurations: smooth bed (blue); rough bed (green); permeable bed (red). OR3 (left) and OR6 (right)

Application to log-fit method to our data leads to the results of Figure 7 for the wave crest phase. The velocity profiles are plotted for different values of $\Delta y / D_{50}=0.20 ; 0.25 ; 0.30 ; 0.35 ; 0.40$.
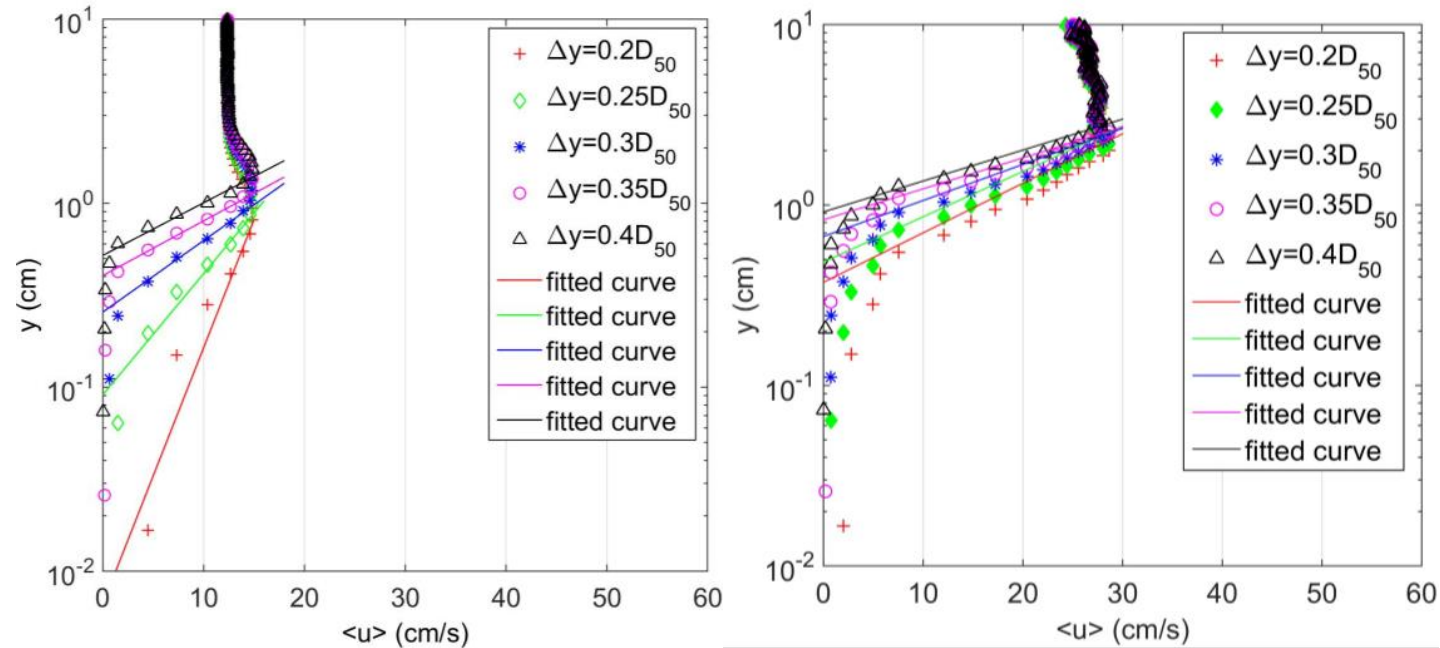

Figure 7 - Semi-log plots of the velocity distribution for five different theoretical-wall locations. OR3 (left) OR6 (right).

It seems that the theoretical wall could lie at $0.25-0.35 D_{50}$ below the top of the spheres over a permeable bed. Such a results can be quite different from a wave to another one. In order to have a fully turbulent flow we used a very large roughness in our experiments (usually evaluated as $2-4 D_{50}$ for rough beds), but a fully rough turbulent flow is not guaranteed for each waves here tested. The wave Reynolds number, $R e=U_{0 M a x} A / v\left(v\right.$ is the fluid kinematic viscosity and $A$ is calculated as $\left.U_{0 M a x} / \sigma\right)$ calculated from the experimental data (see Table 2), was found to be about $10^{3}-10^{4}$. Although this, we try to compare the results obtained for different waves by using a common "true seabed". As a consequence of what observed in Figure 5 and Figure 6 we decide to set the "true seabed" at a location 
deeper with respect to that used in the experiments of Dixen et al. (2008) and suitable for all the waves here analyzed, this being at a distance of $0.3 D_{50}$ below the top of the spheres.

The downward shift of the "true seabed", associated with the presence of a porous bed, leads to values of $k_{s}$ larger $\left(k_{s}=3-6 D_{50}\right)$ than those reported in the literature for a rough impermeable bed. From the knowledge of the friction velocity $u_{*}$, the friction factor $f_{w}$ has been evaluated by means of equation (7) and, hence, the maximum bottom shear stress $\left(\tau_{\max }\right)$ has been calculated as $\tau_{\max }=0.5 f_{w} \rho U^{2}{ }_{\text {oMax }}$ (Jonsson 1967). A larger shear stress is obtained with increase of the Ursell parameter $\left(U_{r}\right)$ but only up to $U_{r}$ of about 40, in agreement with the results about the wave height attenuation over a permeable bed (Corvaro et al. 2010). A further increase of $U_{r}$ leads to a decrease of the Reynolds shear stress.

\begin{tabular}{|c|c|c|c|c|c|c|c|c|c|c|}
\hline Wave & $\begin{array}{l}H_{i} \\
(\mathrm{~m})\end{array}$ & $\begin{array}{l}T \\
\text { (s) }\end{array}$ & $\begin{array}{l}U_{0 M a x} \\
(\mathrm{~cm} / \mathrm{s})\end{array}$ & $\begin{array}{l}A \\
(\mathrm{~cm})\end{array}$ & $\begin{array}{l}R e \\
(-)\end{array}$ & $\begin{array}{l}k_{s} \\
(\mathrm{~cm})\end{array}$ & $\begin{array}{l}u_{*} \\
(\mathrm{~cm} / \mathrm{s})\end{array}$ & $\begin{array}{l}A / k_{s} \\
(-)\end{array}$ & $\begin{array}{l}f_{w} \\
(-)\end{array}$ & $\begin{array}{l}\tau_{\max } \\
\left(\mathrm{N} / \mathrm{m}^{2}\right)\end{array}$ \\
\hline OR2 & 4.4 & 1.5 & 11.60 & 2.77 & $3.21 \times 10^{\wedge} 3$ & 7.02 & 4.20 & 0.39 & 0.26 & 1.76 \\
\hline OR3 & 4.5 & 2.0 & 12.72 & 4.05 & $5.15 \times 10^{\wedge} 3$ & 7.69 & 4.47 & 0.53 & 0.25 & 2.00 \\
\hline OR4 & 8.5 & 2.5 & 26.84 & 10.68 & $2.87 \times 10^{\wedge} 4$ & 16.71 & 9.68 & 0.64 & 0.26 & 9.37 \\
\hline OR5 & 9.3 & 1.5 & 23.06 & 5.50 & $1.27 \times 10^{\wedge} 4$ & 11.84 & 10.01 & 0.46 & 0.38 & 10.03 \\
\hline OR6 & 9.6 & 2.0 & 26.52 & 8.44 & $2.24 \times 10^{\wedge} 4$ & 17.66 & 10.53 & 0.48 & 0.32 & 11.08 \\
\hline OR10 & 11.6 & 2.5 & 35.56 & 14.15 & $5.03 \times 10^{\wedge} 4$ & 22.73 & 8.57 & 0.62 & 0.12 & 7.34 \\
\hline
\end{tabular}

\section{Turbulent components}

Due to the uncertainness on the application of such a method, the shear stress has been evaluated also from the turbulent components $\left\langle-u{ }^{\prime} v{ }^{\prime}\right\rangle$. In Figure 8, Figure 9 and Figure 10 are shown the nondimensional Reynolds shear stresses near the bed for phases close to the wave crest phase (the wave crest phase is displayed in circle), respectively for waves OR2, OR3 and OR6. The shift between the maximum of the wave velocity signal (or surface displacement) and the friction velocity $u_{*}$ is $45^{\circ}$ for the laminar solution. The phase difference in the literature for a real rough bed is smaller than $45^{\circ}$, as obtained by Sleath (1987), Jensen (1989), Dixen et al. (2008). In agreement with what observed in the literature we obtained a phase shift in the range of $10^{\circ}-25^{\circ}$ for a rough bed. For a permeable beds such a phase difference decreases, being about $5^{\circ}-20^{\circ}$. It may be explained by the influence of the flow exchange between the two layers. Inspection of the Reynold shear stress profiles reveals that the turbulent components $\left\langle-u^{\prime} v{ }^{\prime}\right\rangle$ over a permeable beds are larger than those over a rough bed, hence, a larger dissipation occur when wave propagates over a permeable bed.
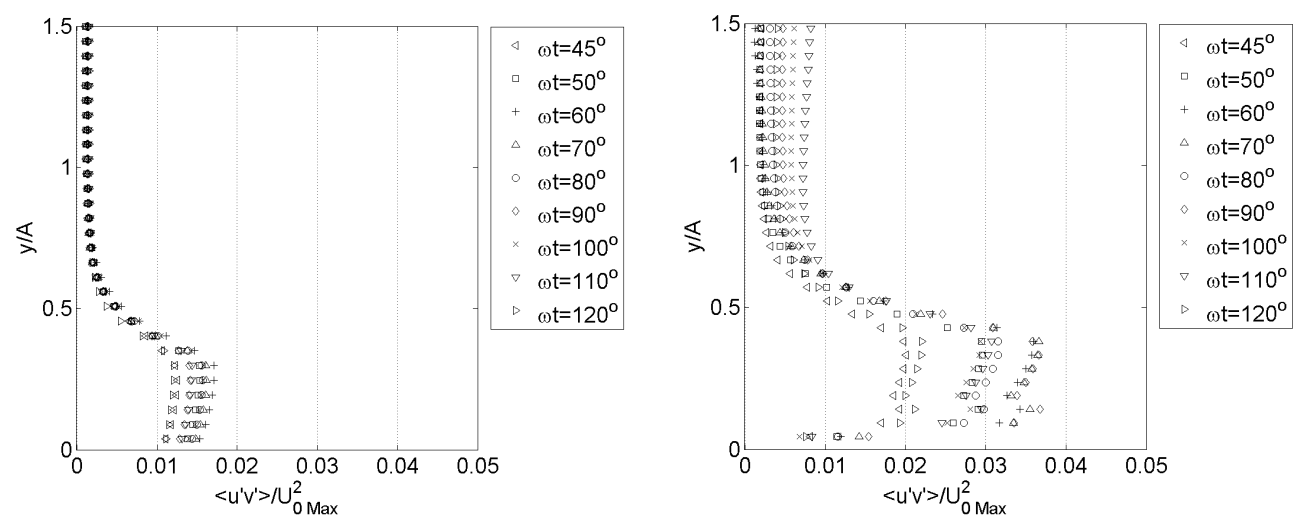

Figure 8 - The non-dimensional Reynold shear stresses for rough bed (left) and permeable beds (right). Wave OR2. The wave crest phase is displayed in circle. 

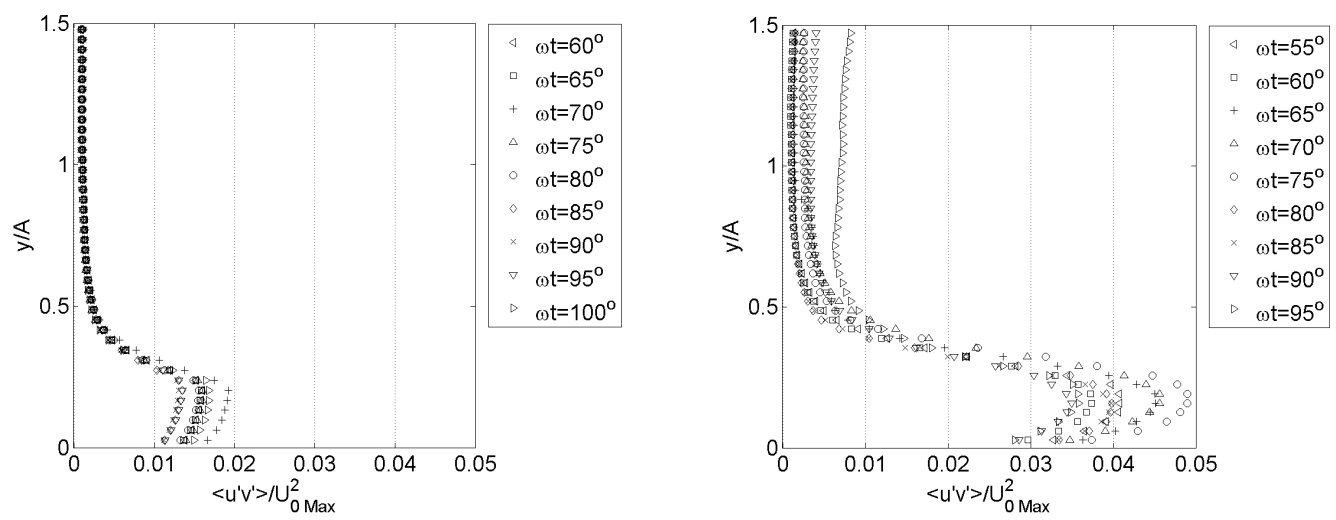

Figure 9 - The non-dimensional Reynold shear stresses for rough bed (left) and permeable beds (right). Wave OR3. The wave crest phase is displayed in circle.
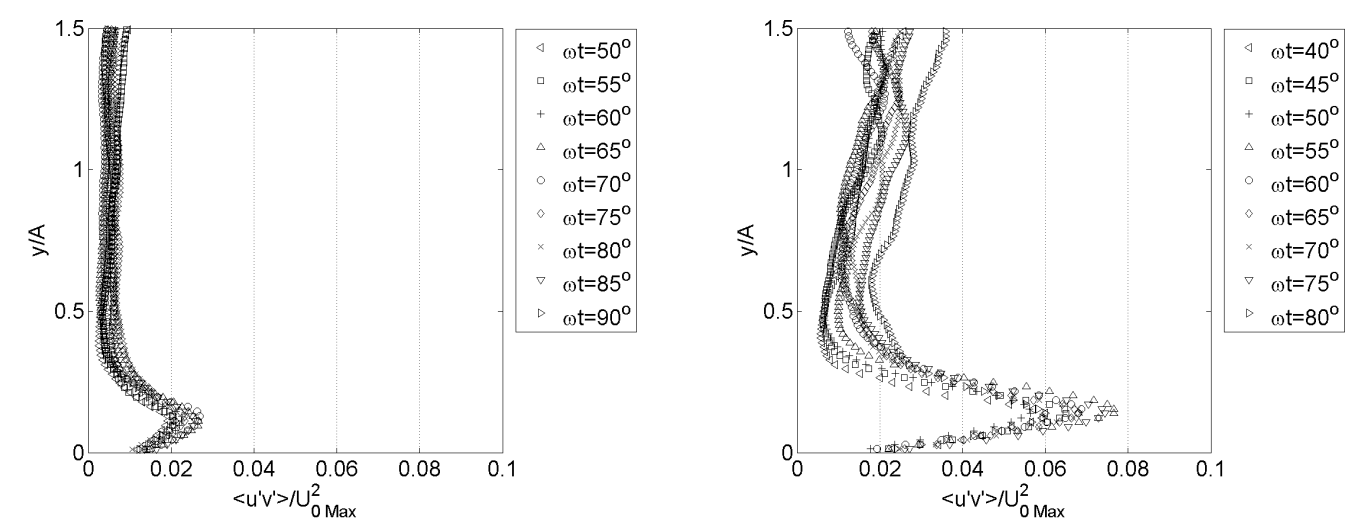

Figure 10 - The non-dimensional Reynold shear stresses for rough bed (left) and permeable beds (right). Wave OR6. The wave crest phase is displayed in circle.

By comparing the maximum shear stress given by the log-fit method and that given by taking the maximum value of the Reynold stress near the bed, we obtain different results. Such a discrepancy has been already observed and explained by Sleath (1986) with the significant fluxes associated by the vortex ejection. Large roughness produces more strongly formed vortices but also more chaotic exchange of fluid from one layer to another. In our experiments the presence of a permeable bed increases further the jets between the porous bed and the flow over it as observed in Corvaro et al. 2014b. Therefore the problem would be even more complicated, even if we believe that our turbulent statistics based on more than 80 waves could be very robust, hence, the comparative analysis can be considered satisfactory.

Moreover, we analyze the effects of a natural permeable beds made of stones. Such an analysis has been here performed only with the latter method, because of the identification of the "true seabed" is even more delicate for irregular bed arrangement. In Figure 11 a comparison of the turbulent components

$\left\langle-u v^{\prime}\right\rangle$ between a single stones layer and a multiple layers has been reported. In agreement with the results about regular beds made of single or multiple layers of spheres, even for natural stones a permeable bed (multiple layers) produces larger turbulent velocity components. In addition a larger turbulent components is observed also by comparing the results about different types of permeable beds: the Reynolds stresses are larger for the irregular permeable bed with respect to the regular ones, hence a stronger wave dissipation is expected for such a type of porous beds. 

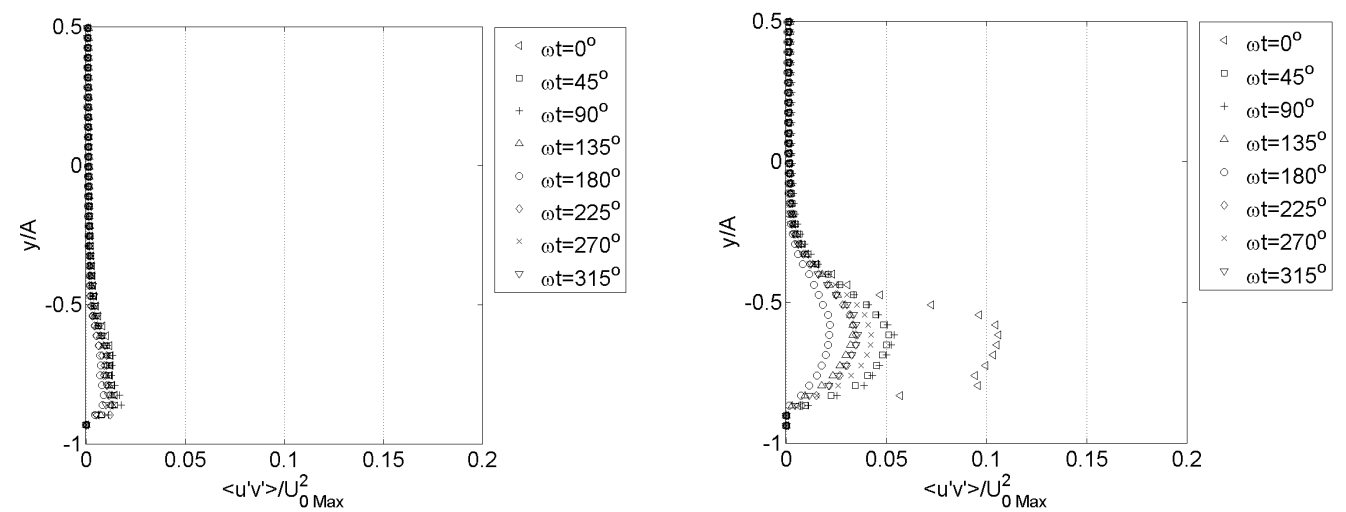

Figure 11 - The non-dimensional Reynold shear stresses for natural rough bed (left) and natural permeable beds (right). Wave OR3.

\section{CONCLUSIONS}

The evaluation of the friction factor $f_{w}$ is important to correlate the behavior of the bottom boundary layer and the wave damping that occurs when a flow propagates over porous beds. The horizontal velocity profiles display the typical behavior of wave forced bottom boundary layers. By using the log-fit method to the mean horizontal velocity profile it is found that the values of the equivalent bottom roughness $k_{s}$ are larger (about 3-6 $D_{50}$ ) than those obtained in the literature for a rough bed. The shear stress obtained by the $\log$-fit method has a non-monotonic behavior with respect to the Ursell parameter, with a maximum at about $U_{r}=40$, in agreement with what found for the wave height attenuation in Corvaro et al. (2010). However, as pointed out in the literature, the application of such a method is delicate, hence we decide to evaluate the shear stress even by means of the turbulent components $\left\langle u v^{\prime}\right\rangle$ '. Larger turbulent components $\left\langle-u^{\prime} v^{\prime}\right\rangle$ have been observed due to permeable beds with respect to rough ones, in agreement with the wave energy dissipation results. The influence of different types of permeable beds is significant: the wave damping induced by the irregular permeable bed is stronger with respect to the regular one. The discrepancy obtained by using two different methods to evaluate the shear stress can be explained in virtue of the very complicate dynamics (see Corvaro et al. 2014b; Miozzi et al. 2015), however a predictive behavior of a permeable bed in term of wave dissipation can be satisfactory achieved. The presents study provides a satisfactory comparative analysis of the flow kinematics over different beds. Further analysis aims to clarify some aspects studied in the present paper.

\section{REFERENCES}

Burcharth, H.F. and Andersen, O.H. 1995. On the one-dimensional steady and unsteady porous flow equations. Coast. Engrg,. 24, 233-257.

Corvaro, S., Mancinelli, A., Brocchini, M., Seta, E. and Lorenzoni, C. 2010. On the wave damping due to a permeable seabed. Coast. Engrg, 57, 1029-1041.

Corvaro, S., Mancinelli, A., Brocchini, M. and Seta, E. 2014a. Flow dynamics on a porous medium. Coast. Engrg, 91, 97-109.

Corvaro, S., Miozzi, M., Postacchini, M., Mancinelli, A. and Brocchini, M. 2014b. Fluid-particle interaction and generation of coherent structures over permeable beds: an experimental analysis. Adv Water Resour 72, 97-109.

Damiani, L., Aristodemo, F., Saponieri, A., Verbeni, B., Veltri, P., Vicinanza, D. 2010. Full scale experiments on a Beach Drainage System: hydrodynamic effects inside the beach. Journal of Hydr. Res., SI Hydralab III.

Damiani, L., Vicinanza, D., Aristodemo, F., Saponieri, A., Corvaro, S. 2011. Experimental investigation on wave set up and nearshore velocity field in presence of a BDS. Journal of Coastal Research, SI 64, 55-59.

Dixen, M., Hatipoglu, F., Sumer, B.M. and Fredsoe, J. 2008. Wave boundary layer over a stone covered bed. Coast. Engrg. 55, 1-20.

Hsiao, S.C., Liu, P.L.-F. and Chen, Y. 2002. Nonlinear water waves propagating over a permeable bed. Proceedings of the Royal Society of London 458, 1291-1322. 
Jensen, B.L., 1989. Experimental Investigation of Turbulent Oscillatory Boundary Layers. Technical University of Denmark, Institute of Hydrodynamics and Hydraulic Engineering, Lygnby, Denmark. Series Paper, 45.

Justesen, P., 1988. Prediction of turbulent oscillatory flow over rough beds. Coast. Eng. 12 (3), $257-$ 284.

Karunarathna, S.A.S.A. and Lin, P., 2006. Numerical simulation of wave damping over porous seabed. Coast. Eng. 53, 845-855.

Lara, J.L., Cowen, E.A., Sou, I.M. 2002. A depth-of-field limited particle image velocimetry technique applied to oscillatory layer flow over a porous bed. Exp. Fluids 33, 47-53.

Losada, I.J., Silva, R. and Losada, M.A., 1996. 3-D non breaking regular wave interaction with submerged breakwaters. Coast. Eng. 28, 229-248.Coast. Engrg. 28, 229-248.

Miozzi, M.; Postacchini, M.; Corvaro, S.; Brocchini, M. 2015. The whole-wavelength description of a wave boundary layer with permeable wall. Exp. Fluids, 56:127, DOI 10.1007/s00348-015-1989-4.

Postacchini, M., Brocchini, M., Corvaro, S., Lorenzoni, C. and Mancinelli, A. 2011. Comparative analysis of sea wave dissipation induced by three flow mechanisms. J. Hydrol. Res. 49(4), 554561.

Sleath, J.F.A. 1987. Turbulent oscillatory flow over rough beds. J. Fluid Mech. 182, 369-409.

Van Gent, M.R.A. 1995. Wave Interaction with Permeable Coastal Structures. (Ph.D Thesis). Delft University.

Wurjanto, A. and Kobayashi, N. 1993. Irregular wave reflection and runup on permeable slope. J Waterw Port C-Asce, 119 (5), 537-557. 